\title{
Building pathways to full encapsulation processes
}

\author{
Abbas Shamsipour ${ }^{1}$, Mohammad Karim Dehghan Manshadi ${ }^{1}$, Danial Khojasteh ${ }^{2}$, Marco \\ Marengo*3 \\ ${ }^{1}$ Department of Mechanical Engineering, Shiraz University, Shiraz, Iran \\ ${ }^{2}$ School of Civil and Environmental Engineering, UNSW, Sydney, Australia \\ ${ }^{3}$ Advanced Engineering Centre, University of Brighton, Brighton, UK \\ ${ }^{*}$ Corresponding author email: m.marengo@brighton.ac.uk
}

\begin{abstract}
Encapsulation, i.e. the coating of tiny solid or liquid particles in a liquid film, has received a growing interest among scholars due to its practical applications, especially in food and pharmaceutical industries. Encapsulation technology is used in coating and controlling drug delivery by protecting the active agents from environmental pressures (e.g., heat, oxygen) and unsought colours/odours. In practice, when dealing with solid particles, this process occurs through impacting liquid droplets of the coating material upon the agent particles. While, in reality, droplet and particle can freely move before and after the collision, a vast majority of the previous efforts to characterize drop-particle impact outcomes have considered that one of the two components is stationary. This study numerically investigates the head-on collisions between freely moving droplet of a glycerine-water solution or synthetic fluids, and freely moving dry spherical particles. In order to achieve this results, a novel 2D axisymmetric Level Set simulation includes fluid-structure interaction (FSI) and an arbitrary Lagrangian-Eulerian (ALE) technique The relative impact velocity and drop-to-particle density ratio are varied across the simulation cases to indicate the importance of variations in momentum. These preliminary results indicate that optimising the momentum is a key factor in achieving a full and stable encapsulation, requiring further research.
\end{abstract}

\section{Keywords}

Droplet, drop-particle collision, momentum transfer, multiphase flow.

\section{Introduction}

To date, many researchers have focused on the droplet impingement on solid flat surfaces [15] due its wide range of applications in inkjet printing, spray cooling, microfluidics, and aerospace engineering [6-11]. In a recent review paper, Khojasteh et al. [12] provided insights into better understanding the dynamics of droplets impacting onto solid flat surfaces (e.g., impact outcomes, importance of surface wettability, contact time). However, in contrast to droplet collision with flat surfaces, little knowledge exists regarding the impact of droplet on spherical surfaces [13]. This is despite the fact that droplet collision with spherical particles has many engineering applications including fluidized catalytic cracking systems, multi-effect desalination evaporators, rain drops impact upon cables and antennas, spray drying, and encapsulation [14].

Among the aforementioned applications, encapsulation has received a surge of interest in recent years due its functionality in food and pharmaceutical industries (e.g., drug delivery and release) by coating covering drug/food bioactive components and protecting them against undesired processes such as high temperature and oxygen levels, unfavourable colours/odours, and light exposure [15]. Encapsulation plays a key role in ensuring the targeted release of bioactive agents inside the human body or within commercial products [15]. A widely applied and cost-effective encapsulation method is spray drying where generated droplets with coating material collide with particles to form a powder with higher 
stability and storage capacity [16]. In a recent review paper, Khojasteh et al., [14] highlighted that a suitable momentum transfer between the droplet and the particle, if optimised, is a key factor which can result in an efficient encapsulation, ensuring a full coverage of particle with the least coating material as well as avoiding the film break-up, splashing, and bouncing of the droplet.

There is a small, but growing body of literature which focused on droplet impact on a dry, stationary particle. Charalampous et al., [17] analysed the head-on collisions of water droplets with stationary particles, and observed three potential outcomes including deposition, formation of a stable crown, and splashing. Khojasteh and colleagues [18, 19] investigated the spreading and rebound patterns of water droplets undergoing collisions with stationary hydrophobic and superhydrophobic spherical surfaces. They proposed a predictive model to estimate the spreading factor (i.e., instantaneous droplet diameter divided by the initial droplet diameter) of water droplets. Liu et al., [20] studied the drop-particle collision by considering the influence of varying contact angle, Weber number, and droplet-to-particle diameter. They reported that the increasing Weber number and the decreasing contact angle would increase the spreading factor. Yoon et al., [21] provided a map of outcomes for head-on droplets impact upon stationary, dry, spherical particles for a range of Weber numbers, wettabilities, and droplet-to-particle diameters. The authors observed two main cases including partial coating (i.e., deposition and rebound) and full coating (i.e., gravity and momentum disintegrations).

The surveyed literature indicates that most of previous efforts to characterise droplet impact dynamics upon particles have focused on stationary particles. However, in reality, droplet and particle can freely move before and after the collision. In a recent study, Yang et al., [22] used 2D numerical simulations to investigate the drop-particle collision when both droplet and particle are freely moving. They noted that when a hydrophobic particle is tested, the droplet and particle would be separated after impact. However, a hydrophilic particle can pass through and split the droplet. Choi et al., [23] investigated the freely moving drop-particle head-on impact with initial contact (i.e., gravitational force was ignored), and identified two main regimes including merging and separation.

To address this knowledge gap, the present study utilises 3D (2D axisymmetric) simulations to examine the influence of drop-particle momentum transfer on the encapsulation outcomes, and how this momentum transfer can be optimised so that a stable coverage around particle is ensured. To this end, relative impact velocity and drop-to-particle density ratio are varied throughout the simulations to achieve a full encapsulation.

\section{Methodology}

In the present study, to model drop-particle collision, three major simulation approaches were coupled together in 2D axisymmetric simulations including level set method (LSM), fluidstructure interaction (FSI), and an arbitrary Lagrangian-Eulerian (ALE) technique. LSM is employed in the model to track the fluid interface as proposed in [24]. Details on LSM are available in previous works along with the fundamental equations [18, 19]. FSI technique [25] is often applied where a deformation in the solid structure is expected due to its interaction with the fluid flow [26, 27] (for more details, see [28]). FSI incorporates an ALE method to take into account the mesh deformation caused by the movement and the impact of droplet and particle [29], and thereby, droplet and particle become freely moving meshes. This integrated method is able to present an accurate prediction of the drop-particle collision dynamics as it considers full underlying physics of the problem, and both droplet and particle are modelled as unrestrained elements during the simulations. Here, a finite-element-based solver package, COMSOL Multiphysics, is employed to couple and solve these set of physical models and the associated equations. Further, an equilibrium contact angle is applied to represent the contact angle of the particle's surface. The predictive accuracy of the model for a droplet impact upon 
a solid stationary particle has been tested, verified, and is available in our previous works [18, $19]$.

\section{Numerical analysis}

Five cases are simulated in this study consisting of both freely moving droplet and particle as illustrated in Figure 1. The properties of glycerine-water solution droplet and dry spherical particles are presented in Table 1. It is assumed that droplet and particle move towards each other with the same velocity so that the relative velocity is sum of both velocities $\left(V_{\text {rel }}=V_{d}+\right.$ $\left.V_{p}\right)$. The ratio of droplet-to-particle diameter is considered as $D^{*}=D_{d} / D_{p}=2$. The system is surrounded with air with a nominal temperature of $293.15 \mathrm{~K}$ at $1 \mathrm{~atm}$ pressure. To highlight the significance of momentum transfer on a successful encapsulation, five simulation cases have been carried out by varying density of particle and relative velocity, as presented in Table 2. Further, a dimensionless number namely Weber number $(W e)$ is used to help in describing drop-particle collision dynamics as follows:

$$
W e=\frac{\rho_{d} V_{r e l}^{2}\left(D^{*} / 2\right)}{\sigma}
$$

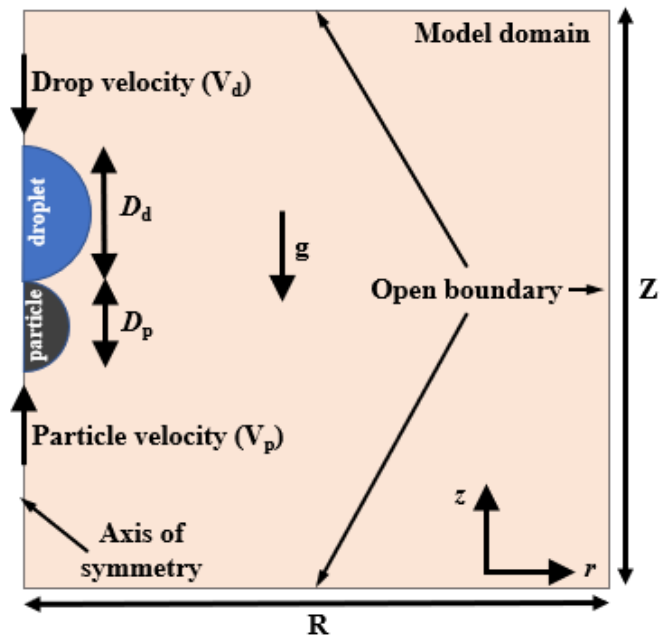

Figure 1. A schematic of model domain and boundary conditions considered in this study.

Table 1. Properties of glycerine-water solution drop and solid particle considered in this study.

\begin{tabular}{c|cccccc}
\hline Material & $\rho\left[\mathrm{kg} / \mathrm{m}^{3}\right]$ & $\sigma[\mathrm{N} / \mathrm{m}]$ & $\mu[\mathrm{Pa} . \mathrm{s}]$ & $\theta\left[{ }^{\circ}\right]$ & $V_{\text {rel }}[\mathrm{m} / \mathrm{s}]$ & $D[\mathrm{~mm}]$ \\
\hline Droplet & 494 & 0.1 & 0.002 & - & $0.65-0.85$ & 3 \\
Particle & $340-617$ & - & - & 40 & & 1.5
\end{tabular}

Table 2. Simulation cases undertaken in this study.

\begin{tabular}{c|cccccc}
\hline Case No. & We & $\rho_{d} / \rho_{p}$ & $V_{\text {rel }}[\mathrm{m} / \mathrm{s}]$ & $\begin{array}{c}(\mathrm{mV})_{d} \\
{\left[\times 10^{-6} \mathrm{~kg} \cdot \mathrm{m} / \mathrm{s}\right]}\end{array}$ & $\begin{array}{c}(\mathrm{mV})_{p} \\
{\left[\times 10^{-6} \mathrm{~kg} \cdot \mathrm{m} / \mathrm{s}\right]}\end{array}$ & $(\mathrm{mV})_{d} /(\mathrm{mV})_{p}$ \\
\hline 1 & 8 & 0.8 & 0.85 & 2.96 & 0.46 & 6.4 \\
2 & 8 & 1.14 & 0.85 & 2.96 & 0.32 & 9.1 \\
3 & 8 & 1.45 & 0.85 & 2.96 & 0.26 & 11.6 \\
4 & 6.25 & 0.8 & 0.75 & 2.62 & 0.41 & 6.4 \\
5 & 4.69 & 0.8 & 0.65 & 2.27 & 0.35 & 6.4
\end{tabular}




\section{Results and Discussion}

Here, the significance of momentum transfer $(m V)$ during freely moving drop-particle collision is studied by changing relative velocity or density of particle. In Case 1, drop and particle have the same velocity of $0.425 \mathrm{~m} / \mathrm{s}$ but in the opposite directions. The density ratio of drop-toparticle is 0.8 and the initial momentum of drop-to-particle is 6.4. The time-lapsed snapshots of the collision for Case 1 are depicted in Figure 2, and the volume fraction (the ratio of volume of particle surrounded in volume of droplet, with volume fraction equals to 0 or 1 indicating that the particle is not or fully covered with droplet, respectively) is shown in Figure 3.

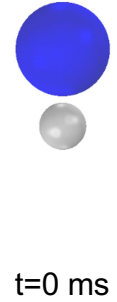

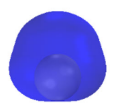

$\mathrm{t}=3 \mathrm{~ms}$

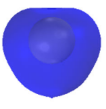

$\mathrm{t}=6 \mathrm{~ms}$

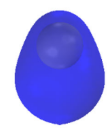

$\mathrm{t}=9 \mathrm{~ms}$

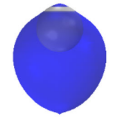

$\mathrm{t}=13 \mathrm{~ms}$

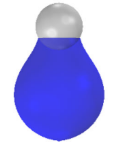

$\mathrm{t}=17 \mathrm{~ms}$

Figure 2. Time-lapsed snapshots of collision for Case 1.

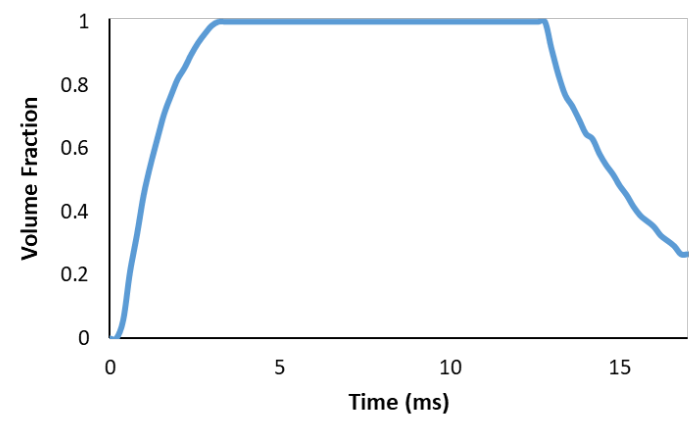

Figure 3. Volume fraction versus time for Case 1.

As shown in these figures, after the collision, the particle stays within the drop and there is a full encapsulation for $9.6 \mathrm{~ms}$. However, at $\mathrm{t}=13 \mathrm{~ms}$ after the impact, the particle starts exiting the droplet. Therefore, the momentum of particle is potentially high and cannot counterbalance the surface tension and viscous forces, and hence, the particle passes through the droplet. Thus, the particle's momentum is reduced in next simulation cases by either decreasing the density of particle (Cases 2 and 3) or reducing the relative velocity of impact (Cases 4 and 5). In case 2, the drop-to-particle density ratio has increased to 1.14 and thereby the associated momentum ratio has increased to 9.1 , while the Weber number is kept constant. The snapshots of impact as well as volume fraction of Case 2 are illustrated in Figure 4 and Figure 5 , respectively.
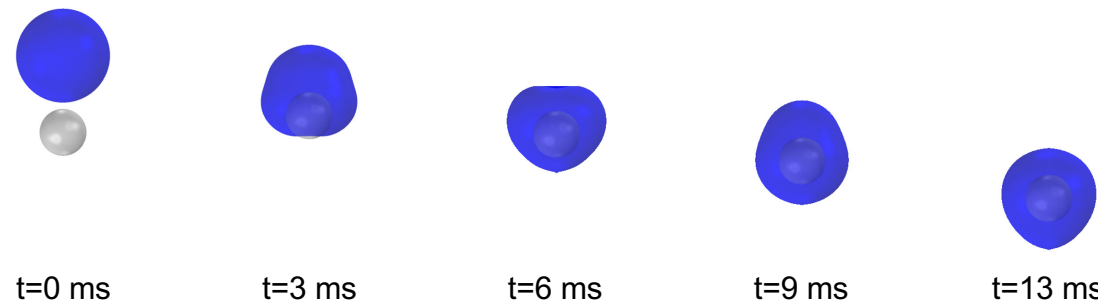

Figure 4. Time-lapsed snapshots of collision for Case 2. 


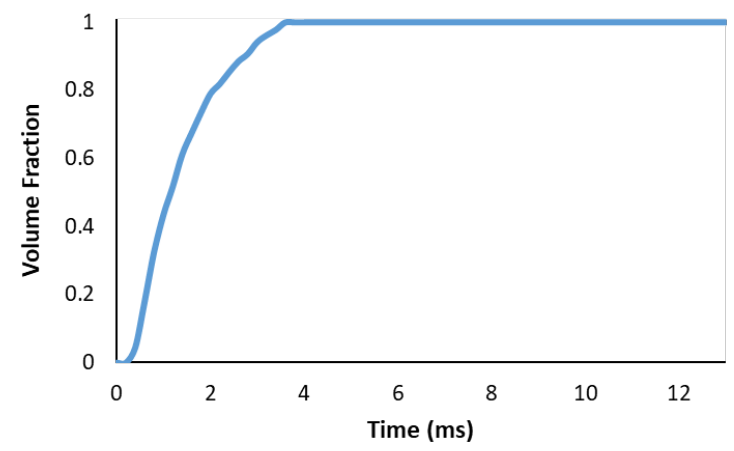

Figure 5. Volume fraction versus time for Case 2.

As is clear from the figures, a full and stable encapsulation has been achieved in Case 2. The reason is that the momentum of the particle is sufficient to offset the surface tension and viscous forces, and simultaneously, not to leave the drop. The influence of a further reduction in particle's momentum is tested in Case 3 where drop-to-particle density ratio is 1.45 and the momentum ratio is 11.6. The similar results as before are reported for Case 3 which are illustrated in Figure 6 and Figure 7. In Case 3, the initial momentum of the particle is insufficient for a full encapsulation. The particle partially enters the droplet up until $4 \mathrm{~ms}$ (with a volume fraction of 0.91 ), and it is then rejected and moves downward.
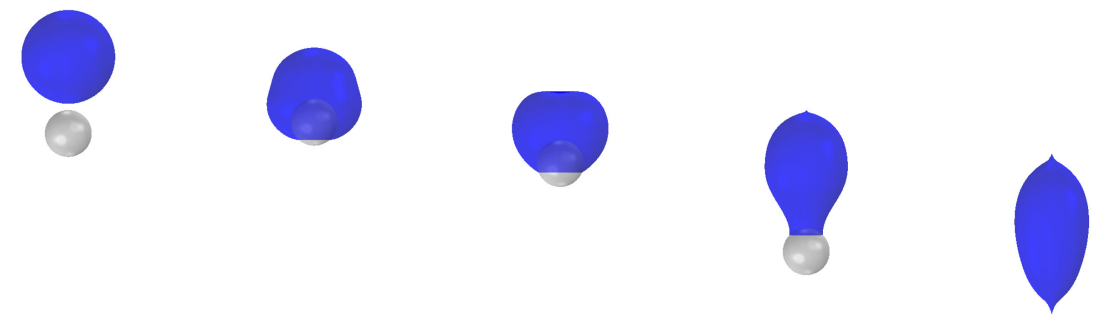

$\mathrm{t}=0 \mathrm{~ms}$

$\mathrm{t}=3 \mathrm{~ms}$

$\mathrm{t}=6 \mathrm{~ms}$

$\mathrm{t}=9 \mathrm{~ms}$

$\mathrm{t}=13 \mathrm{~ms}$

Figure 6. Time-lapsed snapshots of collision for Case 3.

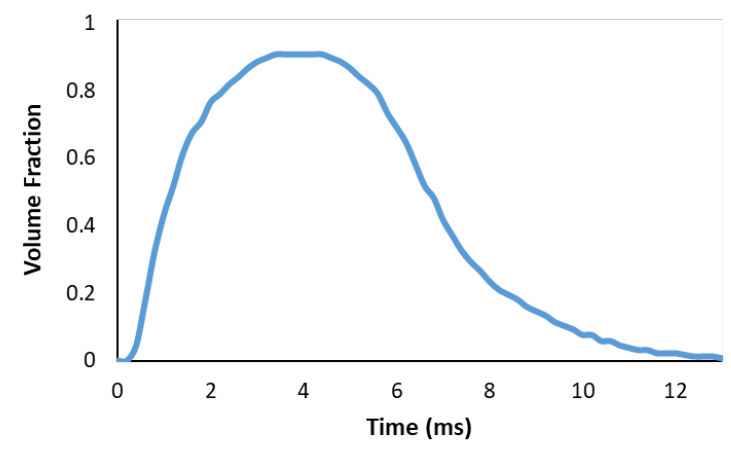

Figure 7. Volume fraction versus time for Case 3.

To examine the influence of momentum reduction by decreasing relative velocity of impact, Case 4 is introduced with a relative velocity of $0.75 \mathrm{~m} / \mathrm{s}$ and a Weber number of 6.25 , while drop-to-particle density ratio and momentum ratio are similar to Case 1 . The corresponding results are indicated in Figure 8 and Figure 9. The results are same as Case 2 where a stable, full capsulation occurs by controlling the momentum transfer during drop-particle collision. In 
comparison to Case 1, the We number and the momentum of drop and particle were reduced, leading to a successful encapsulation.

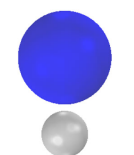

$\mathrm{t}=0 \mathrm{~ms}$

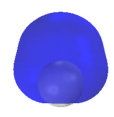

$\mathrm{t}=3 \mathrm{~ms}$

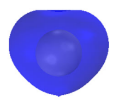

$\mathrm{t}=6 \mathrm{~ms}$

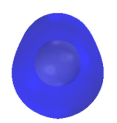

$\mathrm{t}=9 \mathrm{~ms}$

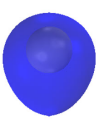

$\mathrm{t}=13 \mathrm{~ms}$

Figure 8. Time-lapsed snapshots of collision for Case 4

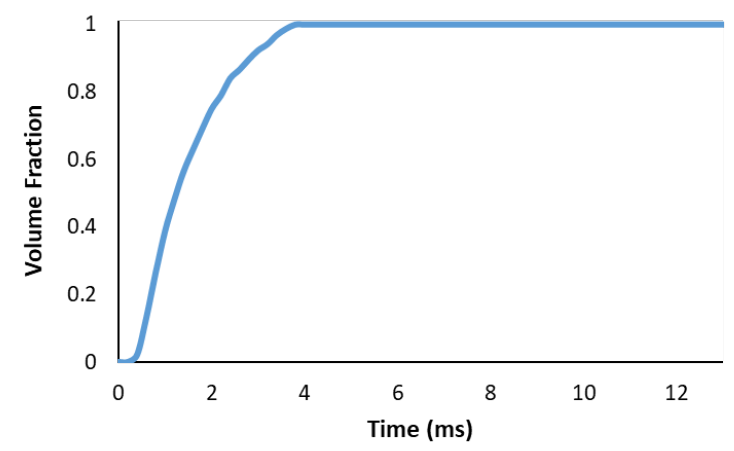

Figure 9. Volume fraction versus time for Case 4

By further reducing the relative velocity of impact to $0.65 \mathrm{~m} / \mathrm{s}$, the We number for Case 5 reaches 4.69, but drop-to-particle density and momentum ratios are same as Case 1 . Results for Case 5 are presented in Figure 10 and Figure 11. The findings are similar to Case 3, where decreasing momentum and We number would result in an ineffective encapsulation. The maximum volume fraction becomes 0.998 at around $7 \mathrm{~ms}$, but then the particle is pushed back and leaves the droplet.

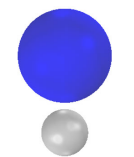

$\mathrm{t}=0 \mathrm{~ms}$

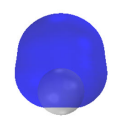

$\mathrm{t}=3 \mathrm{~ms}$
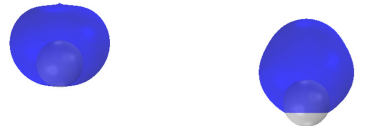

$\mathrm{t}=9 \mathrm{~ms}$

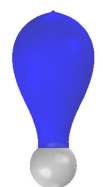

$\mathrm{t}=13 \mathrm{~ms}$

Figure 10. Time-lapsed snapshots of collision for Case 5.

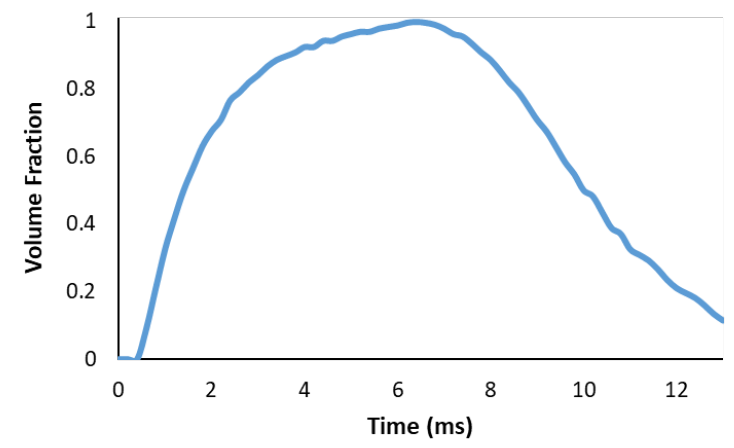

Figure 11. Volume fraction versus time for Case 5. 


\section{Conclusions}

In this study, 3D (2D axisymmetric) simulations cases have been carried out to investigate freely moving drop-particle collision. The key parameters including the relative velocity of impact and density of particle are varied across the simulation cases to highlight the influence of variations in momentum transfer on a full and stable encapsulation. Results show that the full encapsulation with a stable condition would only occur if an appropriate initial momentum are specified to droplet and particle for a given set of physicochemical parameters (density ratio, surface tension, viscosity, and surface wettability). Otherwise, the particle either passes through or is rejected to enter the droplet. Our future work will expand this finding by testing further variations in density and impact velocity as well as viscosity and ratio of drop-to-particle diameter. The physical interpretation of the process in terms of dimensionless numbers is still missing, and it will be part of the future development of the present research.

\section{Nomenclature}

$\begin{array}{ll}\rho & \text { Density }\left[\mathrm{kg} / \mathrm{m}^{3}\right] \\ \sigma & \text { Surface tension }[\mathrm{N} / \mathrm{m}] \\ \mu & \text { Dynamic viscosity of the liquid }[\mathrm{Pa} \mathrm{s}] \\ \theta & \text { Equilibrium contact angle }\left[^{\circ}\right] \\ D & \text { Diameter }[\mathrm{m}] \\ V & \text { Velocity }[\mathrm{m} / \mathrm{s}] \\ W e & \text { Weber number }\end{array}$

\begin{tabular}{ll}
\multicolumn{2}{l}{ Subscripts } \\
$d$ & Drop \\
$p$ & Particle \\
rel & Relative
\end{tabular}

\section{References}

[1] Kamali, R., Khojasteh, D., and Mousavi, S. M., "Newtonian and non-Newtonian droplet impact onto a heated hydrophobic solid surface," Proc. Annual meeting for 24th annual international conference on mechanical engineering-ISME, Yazd, Iran, April, pp. 26-28.

[2] Khojasteh, D., Manshadi, M. K. D., Mousavi, S. M., and Kamali, R., "Droplet impact on superhydrophobic surface under the influence of an electric field," Proc. annual meeting for 3rd Annual International Conference on New Research Achievements in Chemistry \& Chemical Engineering, Tehran, Iran, September.

[3] Khojasteh, D., Manshadi, M. K. D., Mousavi, S. M., Sotoudeh, F., Kamali, R., and Bordbar, A., 2020, "Electrically modulated droplet impingement onto hydrophilic and (super) hydrophobic solid surfaces," Journal of the Brazilian Society of Mechanical Sciences and Engineering, 42(4), pp. 1-11.

[4] Khojasteh, D., Mousavi, S. M., and Kamali, R., 2017, "CFD analysis of Newtonian and non-Newtonian droplets impinging on heated hydrophilic and hydrophobic surfaces," Indian Journal of Physics, 91(5), pp. 513-520.

[5] Sotoudeh, F., Kamali, R., Mousavi, S. M., Karimi, N., Lee, B. J., and Khojasteh, D., 2021, "Understanding droplet collision with superhydrophobic-hydrophobic-hydrophilic hybrid surfaces," Colloids and Surfaces A: Physicochemical and Engineering Aspects, 614, p. 126140.

[6] Antonini, C., Innocenti, M., Horn, T., Marengo, M., and Amirfazli, A., 2011, "Understanding the effect of superhydrophobic coatings on energy reduction in anti-icing systems," Cold Regions Science and Technology, 67(1), pp. 58-67.

[7] Kim, J., 2007, "Spray cooling heat transfer: The state of the art," International Journal of Heat and Fluid Flow, 28(4), pp. 753-767.

[8] Yusof, A., Keegan, H., Spillane, C. D., Sheils, O. M., Martin, C. M., O'Leary, J. J., Zengerle, R., and Koltay, P., 2011, "Inkjet-like printing of single-cells," Lab on a Chip, 11(14), pp. 2447-2454.

[9] Dehghan Manshadi, M. K., Khojasteh, D., Mohammadi, M., and Kamali, R., 2016, "Electroosmotic micropump for lab-on-a-chip biomedical applications," International Journal of Numerical Modelling: Electronic Networks, Devices and Fields, 29(5), pp. 845-858.

[10] Manshadi, M. K., Khojasteh, D., Abdelrehim, O., Gholami, M., and Sanati-Nezhad, A., 2021, "Droplet-based microfluidic platforms and an overview with a focus on application in biofuel generation," Advances in Bioenergy and Microfluidic Applications, pp. 387-406. 
[11] Manshadi, M. K. D., Khojasteh, D., Mansoorifar, A., and Kamali, R., "Efficiency enhancement of ICEK micromixer by a rectangular obstacle," Proc. 3rd annual international conference on new research achievements in chemistry and chemical engineering. Ferdowsi University of Mashhad, Tehran.

[12] Khojasteh, D., Kazerooni, M., Salarian, S., and Kamali, R., 2016, "Droplet impact on superhydrophobic surfaces: A review of recent developments," Journal of Industrial and Engineering Chemistry, 42, pp. 1-14.

[13] Malgarinos, I., Nikolopoulos, N., and Gavaises, M., 2016, "A numerical study on droplet-particle collision dynamics," International Journal of Heat and Fluid Flow, 61, pp. 499-509.

[14] Khojasteh, D., Kazerooni, N. M., and Marengo, M., 2019, "A review of liquid droplet impacting onto solid spherical particles: A physical pathway to encapsulation mechanisms," Journal of industrial and engineering chemistry, 71, pp. 50-64.

[15] Assadpour, E., and Jafari, S. M., 2019, "Advances in spray-drying encapsulation of food bioactive ingredients: From microcapsules to nanocapsules," Annual review of food science and technology, 10, pp. 103-131.

[16] Arpagaus, C., Collenberg, A., Rütti, D., Assadpour, E., and Jafari, S. M., 2018, "Nano spray drying for encapsulation of pharmaceuticals," International Journal of Pharmaceutics, 546(1), pp. 194-214.

[17] Charalampous, G., and Hardalupas, Y., 2017, "Collisions of droplets on spherical particles," Physics of Fluids, 29(10), p. 103305.

[18] Bordbar, A., Taassob, A., Khojasteh, D., Marengo, M., and Kamali, R., 2018, "Maximum spreading and rebound of a droplet impacting onto a spherical surface at low Weber numbers," Langmuir, 34(17), pp. 5149-5158.

[19] Khojasteh, D., Bordbar, A., Kamali, R., and Marengo, M., 2017, "Curvature effect on droplet impacting onto hydrophobic and superhydrophobic spheres," International Journal of Computational Fluid Dynamics, 31(6-8), pp. 310-323.

[20] Liu, X., Zhang, X., and Min, J., 2019, "Maximum spreading of droplets impacting spherical surfaces," Physics of Fluids, 31(9), p. 092102.

[21] Yoon, I., and Shin, S., 2021, "Direct numerical simulation of droplet collision with stationary spherical particle: A comprehensive map of outcomes," International Journal of Multiphase Flow, 135, p. 103503.

[22] Yang, B., and Chen, S., 2018, "Simulation of interaction between a freely moving solid particle and a freely moving liquid droplet by lattice Boltzmann method," International Journal of Heat and Mass Transfer, 127, pp. 474484.

[23] Choi, G., and Shin, S., 2019, "Numerical investigation of freely moving particle-droplet interaction with initial contact," JMST Advances, 1(1), pp. 57-63.

[24] Olsson, E., and Kreiss, G., 2005, "A conservative level set method for two phase flow," Journal of computational physics, 210(1), pp. 225-246.

[25] Khojasteh, D., and Kamali, R., 2017, "Design and dynamic study of a ROV with application to oil and gas industries of Persian Gulf," Ocean Engineering, 136, pp. 18-30.

[26] Krueger, P. S., Hahsler, M., Olinick, E. V., Williams, S. H., and Zharfa, M., 2019, "Quantitative classification of vortical flows based on topological features using graph matching," Proceedings of the Royal Society A: Mathematical, Physical and Engineering Sciences, 475(2228), p. 20180897.

[27] Zharfa, M., Ozturk, I., and Yavuz, M. M., 2016, "Flow Structure on Nonslender Delta Wing: Reynolds Number Dependence and Flow Control," AIAA Journal, 54(3), pp. 880-897.

[28] Zienkiewicz, O. C., Taylor, R. L., and Nithiarasu, P., 2014, "Chapter 13 - Fluid-Structure Interaction," The Finite Element Method for Fluid Dynamics (Seventh Edition), O. C. Zienkiewicz, R. L. Taylor, and P. Nithiarasu, eds., Butterworth-Heinemann, Oxford, pp. 423-449.

[29] Pedrol, E., Massons, J., Dıaz, F., and Aguiló, M., 2018, "Two-Way Coupling FSI Approach to Inertial Focusing Dynamics Under Dean Flow Patterns in Asymmetric Serpentines," preprints. org. 